\title{
Anisotropic effective medium properties from interacting Ag nanoparticles in silicon dioxide
}

\author{
Thiago Menegotto* and Flavio Horowitz \\ Physics Institute and Microelectronics Graduate Program, Universidade Federal do Rio Grande do Sul, \\ Campus do Vale, 15051, Porto Alegre, RS 91501-970, Brazil \\ *Corresponding author: thiagomenegotto@yahoo.com.br
}

Received 9 January 2014; revised 24 March 2014; accepted 24 March 2014; posted 25 March 2014 (Doc. ID 204218); published 25 April 2014

\begin{abstract}
Films containing a layer of Ag nanoparticles embedded in silicon dioxide were produced by RF magnetron sputtering. Optical transmittance measurements at several angles of incidence (from normal to $75^{\circ}$ ) revealed two surface plasmon resonance (SPR) peaks, which depend on electric field direction: one in the ultraviolet and another red-shifted from the dilute $\mathrm{Ag} / \mathrm{SiO}_{2}$ system resonance at $410 \mathrm{~nm}$. In order to investigate the origin of this anisotropic behavior, the structural properties were determined by transmission electron microscopy, revealing the bidimensional plane distribution of Ag nanoparticles with nearly spherical shape as well as the filling factor of metal in the composite. A simple model linked to these experimental parameters allowed description of the most relevant features of the SPR positions, which, depending on the field direction, were distinctly affected by the coupling of oscillations between close nanoparticles, as described by a modified Drude-Lorentz dielectric function introduced into the Maxwell-Garnett relation. This approach allowed prediction of the resonance for light at $75^{\circ}$ incidence from the SPR position for light at normal incidence, in good agreement with experimental observation. (C) 2014 Optical Society of America
\end{abstract}

OCIS codes: (160.1190) Anisotropic optical materials; (160.4760) Optical properties; (240.6680)

Surface plasmons; (310.6860) Thin films, optical properties.

http://dx.doi.org/10.1364/AO.53.002853

\section{Introduction}

Nanostructured materials composed of noble metal nanoparticles have been attractive to the scientific community due to their amazing opportunities of application [1] as, for example, in optical coatings [2], plasmonic sensing [3], and solar cell efficiency improvement [4-6], where optical properties must be well known and understood to allow designing for a specific application [6- $-\underline{8}]$. This implies proper description of the effective medium dielectric function of the nanocomposite layers.

$1559-128 \mathrm{X} / 14 / 132853-07 \$ 15.00 / 0$

(C) 2014 Optical Society of America
Depending on the filling factor of nanoparticles composing a bidimensional layer, the well-known Maxwell-Garnett (MG) effective medium theory $[9,10]$ may lose physical meaning due to the breakdown of the symmetry that was present in the volumetric distribution of nanoparticles, which may lead to anisotropic dipolar interaction among those particles. This limitation in the MG model has been addressed by several authors. Yamaguchi et al. introduced the effective depolarization factor [11]; Granqvist and Hunderi analyzed the role of dipolar interaction considering the depolarization factor [12]; Bedaux and Viegler rigorously introduced the Thin Island Film Theory to describe the electromagnetic interaction between nanoparticles [13]; Barrera et al. presented the idea of the dressed 
polarizability, defined as the ratio between the enhanced dipole moment and the incident electric field [14]; Persson and Liebsch determined the effective polarizabilities of the nanoparticles [15]; Yoo and Park [16] proposed an effective permittivity for ordered structures of nanoparticles with in-plane anisotropy, i.e., nanoparticles disposed in rectangular lattice structures. This permittivity considers the influence of the dipole field of the first neighbor particles.

The MG model, as well as the Drude relation for free electron metals, was originally designed for bulk optical constants valid for metal particles sizes larger than the electron mean free path. For particles smaller than this, interaction of free electrons with nanoparticle walls becomes important. Therefore, additional damping occurs, dependent on the Fermi velocity of electrons in the metal, $v_{F}$, and on the particle radius, $R[\underline{17}, \underline{18}]$ :

$$
\Gamma(R)=\Gamma_{\infty}+A \frac{v_{F}}{R},
$$

where the bulk electron relaxation constant $\Gamma_{\infty}$ is increased due to the scattering of electrons on the nanoparticle surface. The strength of this interaction is given by the phenomenological parameter $A$.

Recently, we proposed an adaptation of the MG model through the Drude relation by using an additional imaginary part for the aforementioned parameter $A$ [19]. This imaginary part of $A$ was responsible for shifting the resonance due to neighbor particle interaction. The complex parameter $A$ was originally introduced to describe changes in plasmon resonance due to nanoparticles adsorbate states [20] and allowed us to attain good fitting to experimental transmittance and reflectance for thin films with interacting nanoparticles [19], although still without quantitative capability to explore directly the physical meaning of $\operatorname{Im}(A)$.

This work goes a step further in the description of the interaction of nanoparticles in layers. We consider a more physical approach to adjust the position of the resonance frequency for thin films with metal nanoparticles. We introduce a parameter related to the ratio between average spacing and nanoparticle size in the layer, as well as to the unshifted plasmon resonance of the single nanoparticle. This parameter directly accounts for the interaction between nanoparticles, which is then included in a couplingextended MG relation. From the proposed solution, we try to grasp the main factors that govern the observed plasmon resonance positions in the measured Ag nanoparticle/silica thin films.

\section{Background Theory}

A. Lorentz Sphere and Interaction Among Nanoparticles in a Planar Structure

The MG model can be used to describe the effective dielectric function of a medium composed of metallic nanoparticles embedded in a dielectric medium
[9,21]. It is a macroscopic representation of the interaction between the electromagnetic wave and the nanostructure in the quasi-static regime.

The concept of the Lorentz local electric field, depicted in Fig. 1, is helpful in understanding the MG model and addressing its limitations to describe properties of layers of nanoparticles. The Lorentz sphere describes an imaginary boundary of arbitrary size. For one particle in the center of this sphere, the medium appears to be homogeneous on the outside, whereas the field contribution of each particle in the Lorentz sphere center has to be summed up individually.

The local field $\mathbf{E}_{\text {local }}$ at the particle in the center of the Lorentz sphere is given by the sum of three terms: the external electric field, $\mathbf{E}_{0}$; the field due to the induced polarization charges at the Lorentz sphere, $\mathbf{E}_{\mathrm{Pol}}$ (not shown in Fig. 1); and the contribution of the dipole electric field of each particle inside the Lorentz sphere to the total electric field at its center, $\mathbf{E}_{\text {dip }}$. For randomly distributed particles in a $3 \mathrm{D}$ system, the sum of dipoles contributing to the local field cancels out on average and the MG effective dielectric function, $\varepsilon_{\text {eff }}$, can be calculated applying the Clausius-Mossoti equation for the polarization of a single particle [17]:

$$
\varepsilon_{\mathrm{eff}}=\varepsilon_{h}\left[1+\frac{3 f\left(\varepsilon_{i}-\varepsilon_{h}\right)}{\left(\varepsilon_{i}+2 \varepsilon_{h}\right)-f\left(\varepsilon_{i}-\varepsilon_{h}\right)}\right]
$$

where $\varepsilon_{i}$ and $\varepsilon_{h}$ are the inclusion particle and host medium dielectric functions, respectively, and $f$ is the volumetric filling factor of particles. For small $f$, Eq. (2) results in a resonance for $\left|\varepsilon_{i}+2 \varepsilon_{h}\right|=0$. If the Drude model is used to describe the dielectric function of the metallic particle and for $\varepsilon_{h}=1$, the surface plasmon resonance (SPR) condition is fulfilled at [15]:

$$
\omega_{\mathrm{SPR}}=\frac{\omega_{p}}{\sqrt{3}},
$$

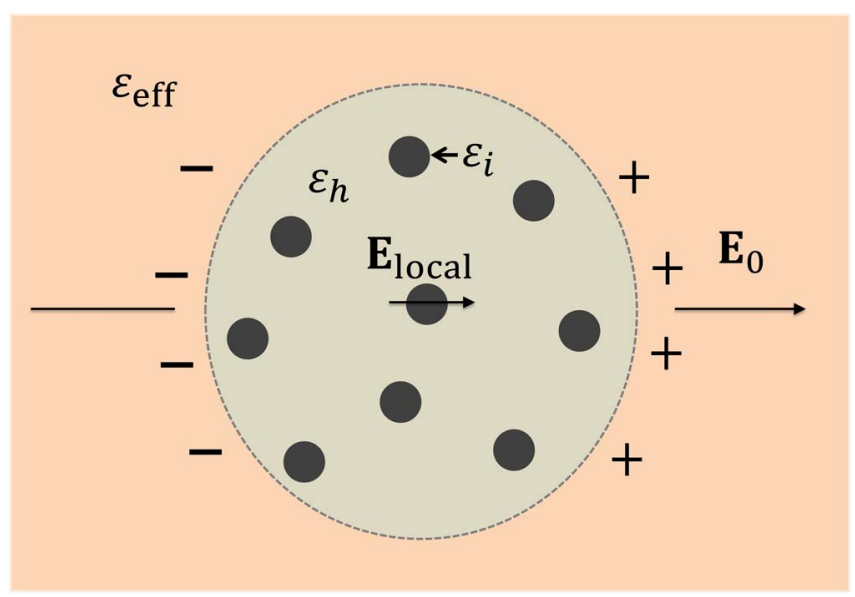

Fig. 1. Lorentz sphere applied to composite media. Explanation of each component can be found in the text. 
where $\omega_{p}$ is the plasma frequency of free electrons in the bulk metal. Equation (3) can be seen as a connection between bulk metal and nanoparticles properties.

For particles distributed in a bidimensional system, such as in thin films containing a layer with nanoparticles, the sum of dipolar electric fields, represented above by $\mathbf{E}_{\text {dip }}$, does not cancel out anymore. Accordingly, one may expect dissimilar contributions to the local electric field in case the applied electric field is parallel or perpendicular to the plane of nanoparticles, and this will result in anisotropic effective dielectric functions.

Persson and Liebsch described the effect of the modified local field in the SPR for a layer of ordered nanoparticles [15]. For a square lattice of characteristic size $a$, in which vertices stand particles of radius $R$ embedded in a medium with $\varepsilon_{h}=1$, there will be two SPR conditions depending on whether the external field $\mathbf{E}_{0}$ is perpendicular or parallel to the layer of nanoparticles. These resonance conditions can be written in terms of the structural parameters ( $a$ and $R$ ) and of the condition of SPR for an isolated nanoparticle [Eq. (3)]:

$$
\begin{aligned}
& \omega_{\mathrm{SPR}, \perp}^{2}=\omega_{\mathrm{SPR}}^{2}\left(1+9.03\left(\frac{R}{a}\right)^{3}\right) ; \\
& \omega_{\mathrm{SPR}, \|}^{2}=\omega_{\mathrm{SPR}}^{2}\left(1-\frac{9.03}{2}\left(\frac{R}{a}\right)^{3}\right),
\end{aligned}
$$

where the factor 9.03 comes from the convergence of the series that parameterizes the square lattice for the inverse cube sum [15].

\section{B. Modeling the Optical Properties of Nanoparticle Layers}

A medium composed of nanoparticles distributed on a bidimensional structure, such as in thin films, might not be expected to follow the dielectric function predicted by the MG model due to the previously described anisotropy of the dipolar interaction with the electric field. Each isolated particle can be treated independently as an oscillating dipole, as electrons of the conduction band of the metal oscillate due to the applied electric field. The resonance frequency of this oscillator is then described by the plasmon resonance. However, in the presence of similar particles in their close vicinity, this oscillation will be influenced by the polarization of those neighbor particles. Figure 2 depicts the cross-sections of bidimensional sets of particles. Based on the work developed by Rechberger et al. [22] for isolated pairs of particles, the interaction between nanoparticles is described by the classical spring-mass coupling between charges in neighbor particles. Considering the case of polarization normal to the plane of nanoparticles the frequency of plasmon resonance for a particle (collective oscillation of electrons within the particle, with spring constant represented by $k_{I}$ ) will be increased by the attractive force among charges in

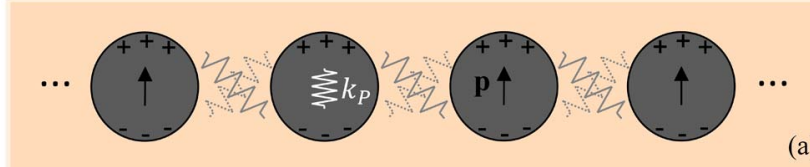

(a)

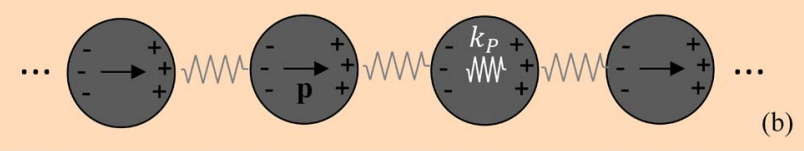

Fig. 2. Cross-section sketches of an infinite bidimensional system of particles representing: (a) polarization perpendicular and (b) polarization parallel to the plane of particles. Schematic springs represent interactions between charges within each nanoparticle (in white) and interactions between charges in neighbor nanoparticles (in gray).

adjacent nanoparticles. This force will effectively correspond to a strengthened spring constant for charge oscillations within each particle [Fig. 2(a)]. In the case of polarization parallel to the plane of particles, the frequency of resonance for a particle will be decreased due to the loosening of the effective spring constant [Fig. 2(b)].

In this work the dielectric function that arises from electrons oscillating in a particle under the influence of neighborhood particles is decoupled of this interaction through the introduction of a modified Drude-Lorentz relation,

$$
m_{e} \frac{\partial^{2} \mathbf{r}}{\partial t^{2}}+m_{e} \Gamma(R) \frac{\partial \mathbf{r}}{\partial t}+\left(k_{I} \pm k_{C}\right) \mathbf{r}=e \mathbf{E}_{0} e^{-i \omega t},
$$

where $m_{e}$ is the electron effective mass; $\Gamma(R)$ describes the phenomenological mean free path limitation for the electrons as shown in Eq. (1); $k_{I}=$ $m_{e} \omega_{\mathrm{SPR}}^{2}$ refers to the attraction of charges within the particle (plasmon resonance for an isolated particle); $k_{c}=m_{e} \omega_{c}^{2}$ is the coupling of oscillating charges of adjacent particles and works as a perturbation for the plasmon resonance of isolated particles; and the term on the right-hand side describes the external force due to the electric field.

In Eq. (6) the choice of the right sign for the coupling parameter $k_{c}$ depends on whether the applied field is perpendicular or parallel to the layer of particles. In accordance with the previous discussion, the positive sign should be applied to the perpendicular polarization and the negative sign to the parallel case. Following Persson and Liebsch description of interaction among nanoparticles in a layer, it is possible to establish an ansatz for $k_{c}$. From Eqs. (4) and (5), the squares of the resonance frequencies are shifted to opposite directions with relation to the free particle plasmon resonance. Furthermore in the perpendicular field case, the squared resonance frequency is displaced by a factor which is twice that of the parallel case. Hence the guess relationship between coupling frequency and structural properties is given by: 


$$
\omega_{c}^{2}=B^{2} \omega_{\mathrm{SPR}}^{2}\left(\frac{R}{a}\right)^{3},
$$

where $B$ is a dimensionless constant that should be equal to $\sqrt{9.03 / 2}=2.1$ for particles uniformly distributed in a square lattice. Additionally, when the medium that surrounds the nanoparticles has dielectric constant $\varepsilon_{h}$, Eq. (3) can be rewritten in the form

$$
\omega_{\mathrm{SPR}}=\frac{\omega_{p}}{\sqrt{1+2 \varepsilon_{h}}} .
$$

In order to modify the MG model, taking into account the interaction among particles since the isolated particle plasmon resonance is already given by the MG-Drude approach, only the additional coupling constant $k_{C}$ needs to be carried on in Eq. (6). Taking the inhomogeneous solution of this equation and using the suitable electromagnetic relations [1] will result in

or

$$
\varepsilon_{i, \perp}(\omega)=1+\frac{f_{c} \omega_{p}^{2}}{\left(2 \omega_{c}^{2}-\omega^{2}\right)-i \Gamma(R) \omega},
$$

$$
\varepsilon_{i, \|}(\omega)=1+\frac{f_{c} \omega_{p}^{2}}{\left(-\omega_{c}^{2}-\omega^{2}\right)-i \Gamma(R) \omega},
$$

respectively, for the dielectric functions perpendicular and parallel to the layer of particles. In these equations the term $f_{c}$ accounts for the oscillator strength.

Equations (9) and (10) can be used to modify the MG relation [Eq. (2)] to include the interaction among particles in a bidimensional system and to describe the anisotropic dielectric properties of nanoparticle layers. Now the extended MG-Drude-Lorentz relation, which accounts for both the plasmon resonance in each particle and the interaction of charges in close particles, becomes

$$
\varepsilon_{\mathrm{eff}, j}=\varepsilon_{h}\left[1+\frac{3 f\left(\varepsilon_{i, j}-\varepsilon_{h}\right)}{\left(\varepsilon_{i, j}+2 \varepsilon_{h}\right)-f\left(\varepsilon_{i, j}-\varepsilon_{h}\right)}\right],
$$

with

$$
\varepsilon_{i, j}(\omega)=1+\frac{f_{c} \omega_{p}^{2}}{\left(j(-1)^{j} \omega_{c}^{2}-\omega^{2}\right)-i \Gamma(R) \omega}, \quad j=1,2 .
$$

In this work Eqs. (11) and (12), with $j=1$ for the electric field parallel to the layer of particles, were applied in conjunction with the characteristic matrix approach [23] for calculation of optical properties of thin films in order to fit the normal incidence transmittance spectra. The structural parameters attained experimentally (average size of particles, average spacing, and filling factor) were used as an initial guess for the calculation. Additionally, the parameters $A, B$, and $f_{c}$ were left to vary. The parameters obtained from the fitting were then applied to the Eqs. (11) and (12) above with $j=2$ to predict the position of the resonance peak for the electric field perpendicular to the layer of particles.

As a final remark in this section, it is interesting to note that one can also clarify the physical significance of $\operatorname{Im}\{A\}$ of [19], where A in Eq. (1) was allowed to be complex, letting the match to measured plasmon-resonance frequency shift from its bulk value. Using Eq. (1) in the Drude-Lorentz relation for an isolated particle, with $A=\operatorname{Re}\{A\}+i \operatorname{Im}\{A\}$ and $r=r_{0} \exp (-i \omega t), \operatorname{Im}\{A\}$ produces a real term that varies linearly with $r$ and can be associated with the coupling of charge oscillations for neighbor particles by comparison with Eq. (ㅁ):

$$
k_{c}=\frac{v_{F}}{R} \omega \operatorname{Im}\{A\},
$$

meaning that the role of $\operatorname{Im}\{A\}$ in the resonance frequency shift is physically due to the interaction among particles.

\section{Experimental}

The films with silver nanoparticles embedded in silicon dioxide $\left(\mathrm{SiO}_{2}\right)$ were produced by $\mathrm{RF}$ magnetron sputtering. The supporting substrates for the depositions were glass and silicon to enable optical transmittance measurements around the visible spectrum and transmission electron microscopy, respectively. A buffer layer of $\mathrm{SiO}_{2}$ was grown simultaneously, prior to the deposition of the Ag particles in both substrates. The deposited layer thicknesses in witness plates with mass equivalent to that of the silver nanoparticles were monitored with a quartz crystal microbalance, allowing precision better than 5\%. Finally an overlayer of $\mathrm{SiO}_{2}$ was deposited to protect the metallic nanoparticles.

Structural properties, size distribution of particles, and average spacing between them were determined from micrographs obtained with a JEM-1220 transmission electron microscope. These were performed in plane-view and in cross-section samples.

The dependence of the interaction between particles on the electric field direction, perpendicular or parallel to the plane of the films, was evaluated by measuring the optical transmittance at angles of incidence ranging from $0^{\circ}$ (normal incidence) to $75^{\circ}$. These measurements were performed with a Cary 5000 spectrophotometer using polarized light to well separate the components of interaction between particles in accordance with the discussion of the previous section.

\section{Results and Discussion}

Figure 3 shows the measured relative transmittance for a sample with particles of silver buried in silicon dioxide. These measurements were taken at several angles of incidence from normal incidence with polarized light either parallel (p) or perpendicular (s) 

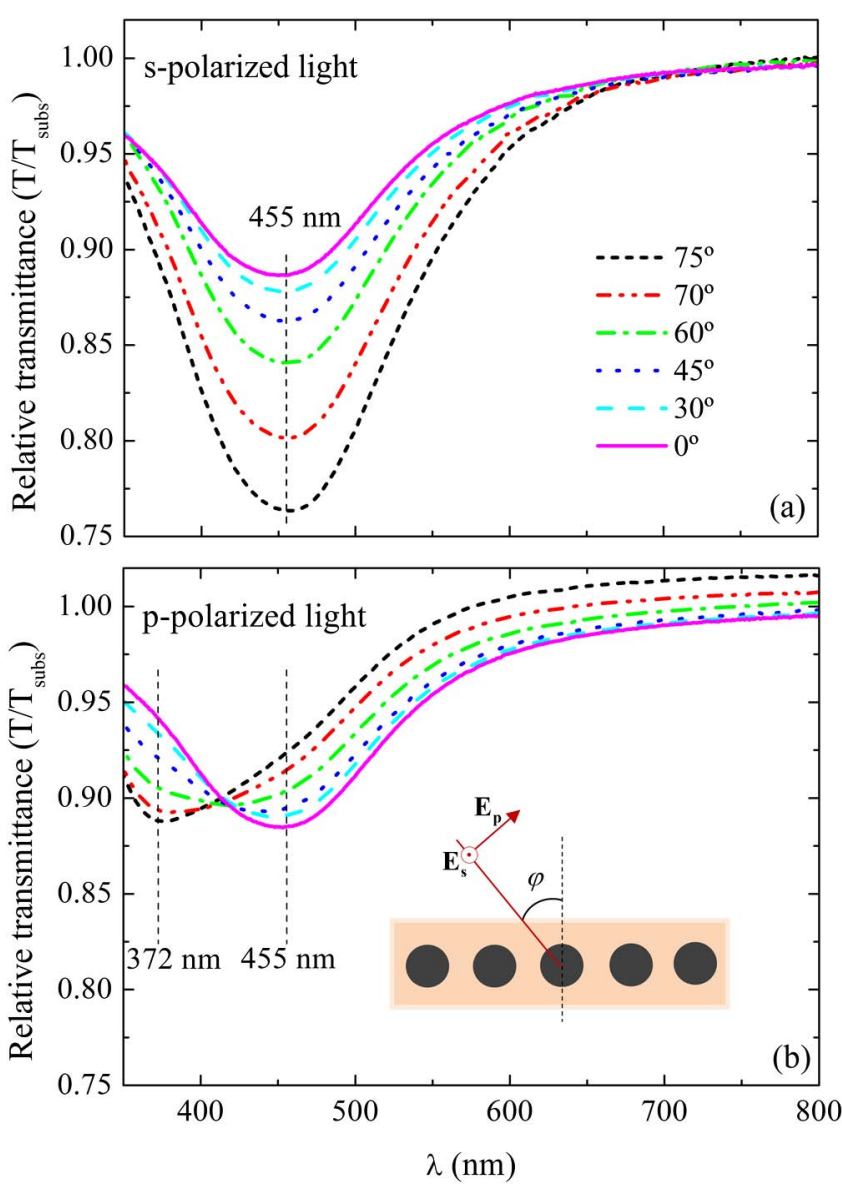

Fig. 3. Experimental transmittance spectra of the nanoparticle thin film on glass, relative to that of the uncoated substrate, for (a) s-polarized light and (b) p-polarized light. The transmittance of the bare substrate at the same angle of incidence and polarization direction was used as reference. The schematic profile at the inset presents the orientation of the electric field with respect to the plane of incidence.

to the plane of incidence. In order to compare the results each measurement was normalized by the transmittance of the bare substrate at the same angle of incidence and polarization direction.

For s-polarized light, Fig. 3(a) depicts the increase of the absorbance peak at $45 \overline{5 \mathrm{~nm}}$ (valley of transmittance) with an increasing angle of incidence. This behavior can be understood simply, as verified by straightforward simulations, by the Fresnel-related reduction of transmitted light for this polarization as the angle of incidence increases. Figure 3(b) shows the behavior of the relative transmittance for p-polarized light. In this case the peak at $455 \mathrm{~nm}$, present also in these spectra, is increasingly smeared out by the increase of the angle of incidence, as the peak at $372 \mathrm{~nm}$ grows. Also for p-polarized light the transmittance of the substrate with film start to become higher than the trasmittance of the bare substrate $\left(T / T_{\text {subs }}>1\right)$ at the red end of the spectrum, where the film exhibits dielectric behavior.

The component of electric field orthogonal to the plane of particles increases as the angle of incidence increases for p-polarized light [see inset of Fig. 3(b)].
In this case the behavior of the film layer with nanoparticles is anisotropic and presents two plasmon resonances, one related to the field parallel and another related to the field perpendicular to the plane of particles, which in the experimental results of Fig. 3(b) are at 455 and $372 \mathrm{~nm}$, respectively. It can be also observed that for s-polarized light, the electric field is always parallel to the plane of particles regardless of the angle of incidence, and this explains why the SPR peak remains at $455 \mathrm{~nm}$ in Fig. 3(a).

The size distribution of the particles was determined by transmission electron microscopy. Figure 4 shows a plane-view micrograph of the produced sample. The average size of the nanoparticles was $8 \mathrm{~nm}$.

The filling factor of particles in the film was estimated to be $15 \%$ from the measured mass thickness of the deposited $\mathrm{Ag}(1.2 \mathrm{~nm})$ and the average particle diameter perpendicular to the film (also $8 \mathrm{~nm}$, obtained from the cross-section micrographs). In order to apply the proposed model, the distance between particles was also calculated. This was achieved by considering each particle in the center of a box with height given by its average diameter and sides given by the average spacing. Note also that the spacing estimate is tied to the filling factor. The result is a spacing of $15 \mathrm{~nm}$, which is satisfactory if one considers the particles being arranged in a square lattice array.

An estimate for the plasmon resonance of isolated nanoparticles of $\mathrm{Ag}$ embedded in $\mathrm{SiO}_{2}$ is also needed. This estimate can be obtained from Mie theory, but due to the permanent charge transfer from within the particles to their surface and the consequent reduction on the density of free electrons available for the plasmon, the results obtained from experiment are different from those of Mie theory. A particularly

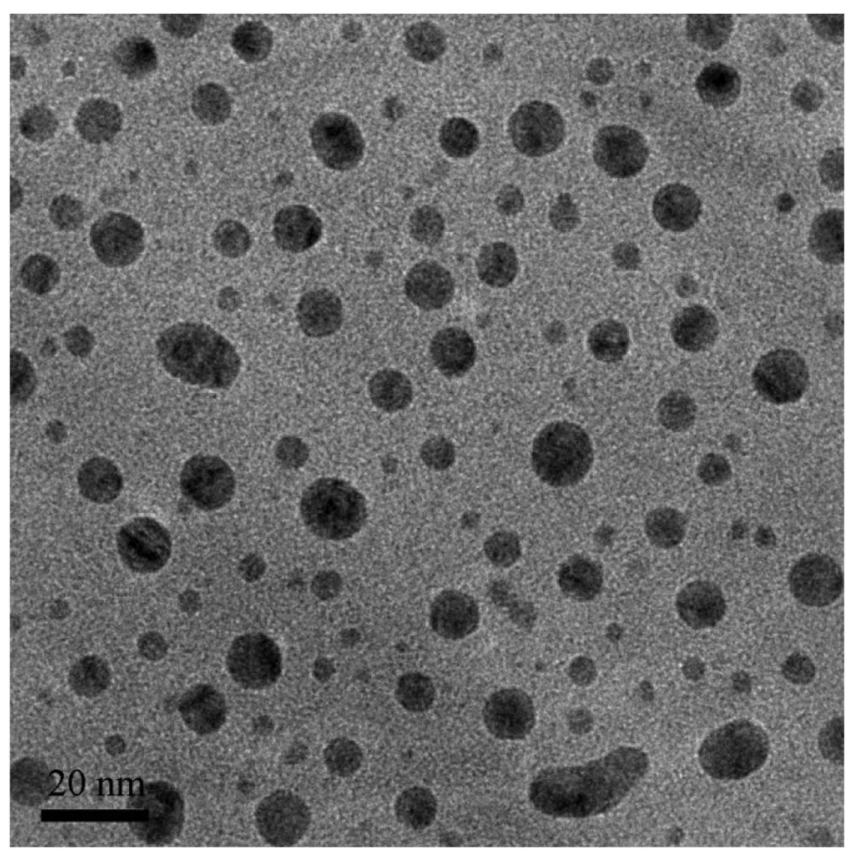

Fig. 4. Plane-view micrograph of the nanoparticle thin film. 
interesting experimental result for the position of the plasmon resonance for narrow-sized, diluted distribution of silver nanoparticles embedded in several dielectric materials was published by Kreibig and coworkers [24]. For $2 \mathrm{~nm}$ diameter Ag nanoparticles in $\mathrm{SiO}_{2}$, the calculated plasmon resonance is $3 \mathrm{eV}$ $(\sim 410 \mathrm{~nm})$, whereas the measured value is $2.65 \mathrm{eV}$ $(\sim 470 \mathrm{~nm})$ [24].

Since the plasmon resonance position is proportional to the square root of the free electron density, it can be expected that about $20 \%$ of the free charge is transferred to (and trapped in) the nanoparticle surface for a $2 \mathrm{~nm}$ diameter $\mathrm{Ag}$ nanoparticles embedded in $\mathrm{SiO}_{2}$, as compared to the bulk silver free electron density $\left(\rho_{\text {bulk }}\right)$. This estimate can be obtained by considering the difference of the squared calculated and experimental resonances, as given above, and their proportionality to the relative variation of the free electron density, $E_{\text {calc }}^{2}-E_{\text {exp }}^{2}=E_{\text {calc }}^{2} \Delta \rho / \rho_{\text {bulk }}$. The permanent charge transfer is also proportional to the ratio of the surface area to the volume of a particle, which means that the effect decreases with the inverse of the radius. In this way it can be expected that the free charge transferred to the surface of the nanoparticle will be $1 / 4$ for $8 \mathrm{~nm}$ when compared to $2 \mathrm{~nm} \mathrm{Ag}$ nanoparticles in $\mathrm{SiO}_{2}$, i.e., about $5 \%$ of the free electrons are now trapped. This leads to a SPR around $2.9 \mathrm{eV}(425 \mathrm{~nm})$ for $8 \mathrm{~nm}$ isolated $\mathrm{Ag}$ nanoparticles embedded in silica.

Once the structural and optical parameters are described, the model presented can be applied to the transmittance results. Figure $\underline{5}$ shows in a continuous curve, the fit for the normal transmittance data using $j=1$ in Eqs. (11) and (12) with absorption peak at $455 \mathrm{~nm}$. The inset presents the best model fitting parameters whose detailed description is given below.

Applying the parameters shown in Fig. $\underline{5}$ to the equations describing the interaction between nanoparticles with the electric field perpendicular

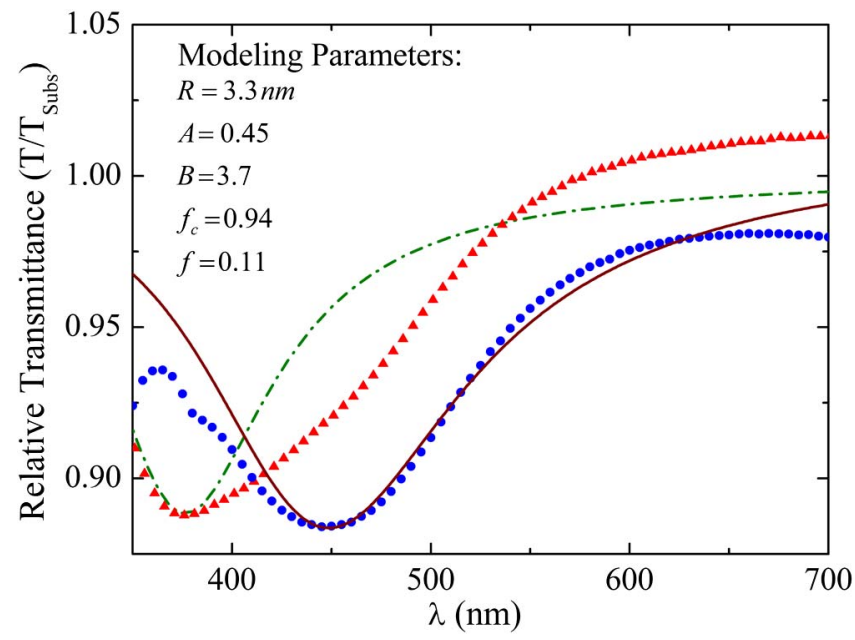

Fig. 5. Relative transmittance data and model fitting for normal incidence (solid line, circles) and $75^{\circ}$ tilted incidence for p-polarized light (dashed line, triangles). to the plane of the film, $j=2$ in Eqs. (11) and (12), one can predict the position of the second absorption peak at $372 \mathrm{~nm}$, as can be observed in the dasheddotted line of Fig. $\underline{5}$ for transmittance at $75^{\circ}$ incidence for $p$-polarized light. This provides evidence of the consistency and accuracy of the proposed approach.

The average radius attained in the fitting presented in Fig. $\underline{5}$ is slightly smaller than the experimental values (3.3 versus $4 \mathrm{~nm}$ ), although well within the size distribution of particles. This can be a consequence of the oxidation of the nanoparticle surroundings, since the film transmittance spectrum was measured a few months after sample preparation. That decrease in average particle radius, which was considered in the film thickness, also provides an explanation for the filling factor determination of 0.11 instead of the 0.15 experimental value. The relaxation parameter $A$, taken as a real number, is related to the width of the resonance curve, and is tied to the particle size via Eq. (1). Since bigger particles contribute more to the resonance peak in a broad size distribution, the value of $A$ from the experiment modeling is of the same order but tends to be smaller than that theoretically predicted in [17] for monosized particles. The oscillator strength parameter $f_{c}$ was 0.94 , which is consistent with the reduction of about $5 \%$ in the free charges described previously. The $B$ parameter value of 3.7 obtained by modeling is influenced by the ratio of particle size to lattice parameter and their influence in Eq. (7) for $\omega_{c}$. Even though the approximate assumption of regular distribution of particles seems to be strong in this case, the positions of both SPRs could be simultaneously matched with the coupling-extended effective medium model.

\section{Conclusion}

In the Ag nanoparticles/silica films produced by $\mathrm{RF}$ magnetron sputtering, transmission electron microscopy revealed a bidimensional plane distribution of Ag particles with nearly spherical shape, average diameter of $8 \mathrm{~nm}$ and average inter-particle spacing of $15 \mathrm{~nm}$. From the equivalent Ag mass thickness measured during deposition, a particle filling factor around 15\% was determined. These experimental parameters and the assumption that the two SPRs were related to the light electric field parallel or perpendicular to the $\mathrm{Ag}$ particle plane allowed for a simple model that could grasp the most relevant system features to its SPR positions. These, depending on the field direction, could be seen as distinctly affected by the coupling of oscillations between neighbor nanoparticles as described by a modified Drude-Lorentz dielectric function, Eq. (6), introduced into a coupling-extended $\mathrm{MG}$ relation in Eqs. (11) and (12) to account for the dependence on frequency and on field orientation. As a consequence, from the SPR position for light at normal incidence, the resonance for light at $75^{\circ}$ incidence 
could be predicted in good agreement with experimental data.

The results show the SPR dependence on polarization shifted from the dilute $\mathrm{Ag} / \mathrm{SiO}_{2}$ system resonance position to the red and to the ultraviolet, and are encouraging for further application to the proposed approach on other metal nanoparticle-rich dielectric thin films.

We are grateful for the support from Conselho de Desenvolvimento Científico e Tecnológico (CNPq) and Coordenação de Aperfeiçoamento de Pessoal de Nível Superior (CAPES), as well as for the UFRGS Microscopy Center's assistance in the transmission electron microscopy measurements.

\section{References}

1. S. A. Maier, Plasmonics: Fundamentals and Applications (Springer, 2007), p. 223.

2. M. Held, O. Stenzel, S. Wilbrandt, N. Kaiser, and A. Tünnermann, "Manufacture and characterization of optical coatings with incorporated copper island films," Appl. Opt. 51, 4436-4447 (2012).

3. K. Lee and M. A. El-sayed, "Gold and silver nanoparticles in sensing and imaging: sensitivity of plasmon response to size, shape, and metal composition," J. Phys. Chem. B 110, 19220-19225 (2006).

4. F. J. Beck, S. Mokkapati, A. Polman, and K. R. Catchpole, "Asymmetry in photocurrent enhancement by plasmonic nanoparticle arrays located on the front or on the rear of solar cells," Appl. Phys. Lett. 96, 033113 (2010).

5. F. J. Beck, A. Polman, and K. R. Catchpole, "Tunable light trapping for solar cells using localized surface plasmons," J. Appl. Phys. 105, 114310 (2009).

6. M. A. Green and S. Pillai, "Harnessing plasmonics for solar cells," Nat. Photonics 6, 130-132 (2012).

7. D. Duche, P. Torchio, L. Escoubas, F. Monestier, J. Simon, F. Flory, and G. Mathian, "Improving light absorption in organic solar cells by plasmonic contribution," Sol. Energy Mater. Sol. Cells 93, 1377-1382 (2009).

8. H. A. Atwater and A. Polman, "Plasmonics for improved photovoltaic devices," Nat. Mater. 9, 205-213 (2010).
9. J. C. M. Garnett, "Colours in metal glasses and in metallic films," Philos. Trans. R. Soc., A 203, 385-420 (1904).

10. J. C. M. Garnett, "Colours in metal glasses, in metallic films, and in metallic solutions-II," Philos. Trans. R. Soc., A 205, 237-288 (1906).

11. T. Yamaguchi, S. Yoshida, and A. Kinbara, "Effect of the dipole interaction between island particles on the optical properties of an aggregated silver film," Thin Solid Films 13, 261-264 (1972).

12. O. Granqvist and C. G. Hunderi, "Optical properties of ultrafine gold particles," Phys. Rev. B 16, 3513-3534 (1977).

13. D. Bedeaux and J. Vlieger, Optical Properties of Surfaces, 2nd ed. (Imperial College, 2004).

14. R. G. Barrera, M. del Castillo-Mussot, and G. Monsivais, "Optical properties of two-dimensional disordered systems on a substrate," Phys. Rev. B 43, 13819-13826 (1991).

15. B. N. J. Persson and A. Liebsch, "Optical properties of two-dimensional systems of randomly distributed particles," Phys. Rev. B 28, 4247-4254 (1983).

16. S. Yoo and Q.-H. Park, "Effective permittivity for resonant plasmonic nanoparticle systems via dressed polarizability," Opt. Express 20, 16480-16489 (2012).

17. U. Kreibig and M. Vollmer, Optical Properties of Metal Clusters (Springer, 1995), p. 532.

18. U. Kreibig and C. V. Fragstein, "The limitation of electron mean free path in small silver particles," Zeitschrift für Physik 224, 307-323 (1969).

19. T. Menegotto, M. B. Pereira, R. R. B. Correia, and F. Horowitz, "Simple modeling of plasmon resonances in $\mathrm{Ag} / \mathrm{SiO}_{2}$ nanocomposite monolayers," Appl. Opt. 50, C27-C30 (2011).

20. A. Pinchuk, U. Kreibig, and A. Hilger, "Optical properties of metallic nanoparticles: influence of interface effects and interband transitions," Surf. Colloid Sci. 557, 269-280 (2004).

21. R. J. Gehr and R. W. Boyd, "Optical properties of nanostructured optical materials," Chem. Mater. 8, 1807-1819 (1996).

22. W. Rechberger, A. Hohenau, A. Leitner, J. R. Krenn, B. Lamprecht, and F. R. Aussenegg, "Optical properties of two interacting gold nanoparticles," Opt. Commun. 220, 137-141 (2003).

23. H. A. Macleod, Thin-film Optical Filters, 3rd ed. (CRC Press, 2001).

24. U. Kreibig, G. Bour, A. Hilger, and M. Gartz, "Optical properties of cluster-matter: influences of interfaces," Phys. Status Solidi A 175, 351-366 (1999). 